\title{
Why Should We Contemplate on Gender Difference in Functional Gastrointestinal Disorders?
}

\author{
Jaehoon Jahng and Yong Sung Kim* \\ Department of Gastroenterology, Wonkwang University Sanbon Hospital, Wonkwang Digestive Disease Research Institute, Gunpo, Gyeonngi- \\ do, Korea
}

Article: Chronic early-life stress in rat pups alters basal corticosterone, intestinal permeability, and fecal microbiota at weaning: influence of sex

Moussaoui N, Jacobs JP, Larauche $\mathrm{M}$, et al

(J Neurogastroenterol Motil 2017;23:135-144)

Even without taking epidemiologic studies into consideration, gastroenterologists naturally get to know from their clinical practice that irritable bowel syndrome (IBS) occurs more often in women than men. This gender difference also should be considered in other functional gastrointestinal disorders (FGIDs) such as gastroesophageal reflux disease. ${ }^{1}$ However, preclinical animal research has been performed mainly on male animals, and sometimes reporting on the sexual difference of animal subjects was missed. It is particularly problematic in those studies investigating the pathophysiology of FGIDs, which manifest differently between male and female patients. When we looked into the sexes of experimental animals in preclinical research articles published in the Journal of Neurogastroenterology and Motility during 2010-2016, male animals were exclusively used and only $10.3 \%$ of studies used both sexes. Surprisingly, $17.3 \%$ of studies did not even report the sex of the experimental animal subjects (Figure). Major granting organizations including NIH is now encouraging that sex should be considered as a biological variable, and requiring sex and gender analysis from a basic study to a clinical trial. ${ }^{2,3}$

In accordance with this recent recommendation and necessity,
Moussaoui et al ${ }^{4}$ not only have shown the importance of postnatal care for the development of intestinal permeability and intestinal microbiota in the suckling period, but also emphasized the sex difference in stress response in the postnatal period. Female rats exposed to limited nesting stress (LNS) showed more prominent stress response such as increase in corticosterone levels and adrenal weights compared to male rats. Moreover, increase in intestinal permeability by LNS also was higher in female rats than male rats. When we translate these findings into clinical practice for patients, we have to interpret the concept of sex difference in animals to gender difference in humans.

By definition, the "sex" means a human's biological classification based on their reproductive systems and functions that derived from chromosomal type or hormones, whereas "gender" means cultural attitudes and behaviors associated with a person's sex stereotypes that shaped masculinity and femininity. ${ }^{5,6}$ In addition to biological effects of sex such as hormonal factors and genetic differences in animal studies, other gender-linked factors including psychosocial behaviors related to stress, mental well-being, gender roles, and the experience of sexual abuse have an important role in

Received: December 6, 2016 Revised: None Accepted: December 6, 2016

(.) This is an Open Access article distributed under the terms of the Creative Commons Attribution Non-Commercial License (http://creativecommons. org/licenses/by-nc/4.0) which permits unrestricted non-commercial use, distribution, and reproduction in any medium, provided the original work is properly cited.

*Correspondence: Yong Sung Kim, MD, PhD

Department of Gastroenterology, Wonkwang University Sanbon Hospital, Sanbon-ro 321, Gunpo, Gyeonggi-do 15865, Korea Tel: +82-31-390-2975, Fax: +82-31-398-2223, E-mail: wms89@hanmail.net 

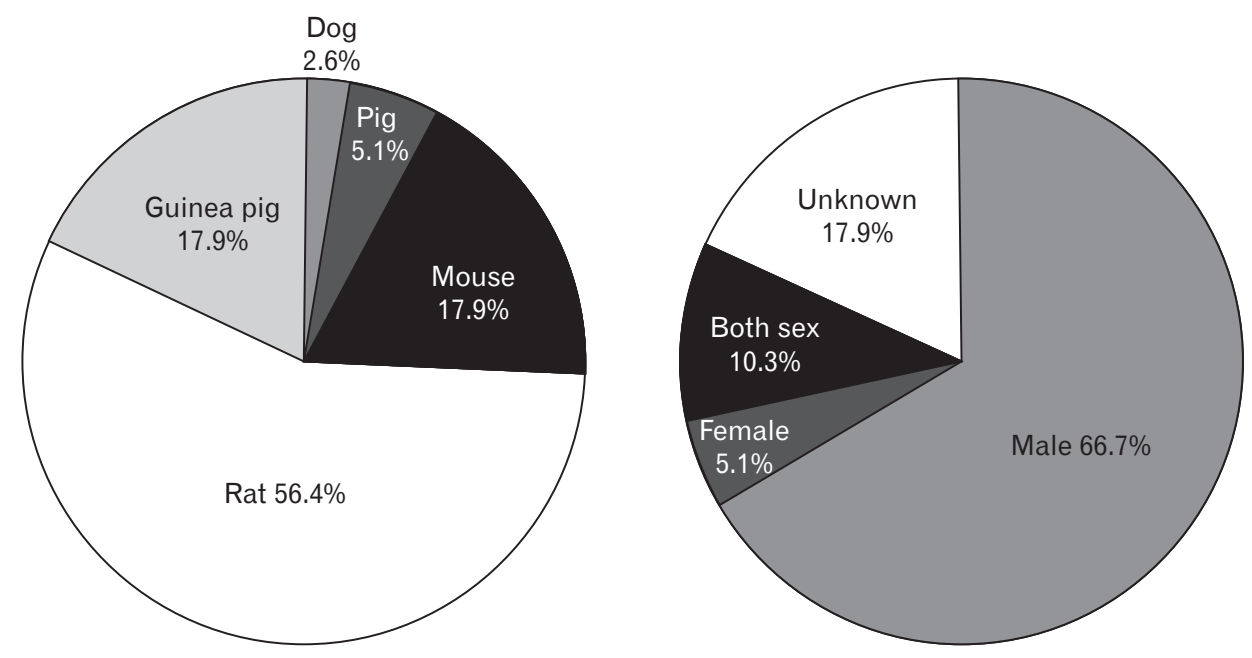

Figure. Species and sex distribution of experimental animal in preclinical research published in the Journal of Neurogastroenterology and Motility from 2010 to 2016. the pathogenesis of IBS. ${ }^{7}$ Therefore, the fundamental difference between male and female animals found in preclinical research could be augmented between men and women in real practice, especially by the prejudice for gender role in different cultures and societies. For example, abdominal bloating impact differently on symptomatology of men and women in the modern society, which has the prejudice that slender women are more beautiful. Unlike men with bloating, women with bloating may usually be concerned about the effect of distention on their appearance leading psychological distress as well as their physical symptom. ${ }^{8}$ Recent research trends have shown that many organic diseases have been re-analyzed according to sex and gender. ${ }^{5}$ Furthermore, gender issue is utmost importance in FGIDs than other organic diseases, and we have many unanswered questions concerning gender differences in FGIDs. In addition, we must not forget that these sexual differences in FGIDs are most prominent in subjects exposed to early life stress than adulthood, at least in rats, according to Moussaoui et al. ${ }^{4}$ Intervention of early life stress in animals or human subjects could potentially benefit from developing FGIDs including IBS through cessation of perturbation and stabilization of intestinal microbiota.

Fortunately, the revised Rome IV criteria emphasized the effect of culture and gender and tried to appropriately assess these issues in clinical studies with different languages, leading to meaningful interpretations. Now is the time for both basic scientists and clinical researchers in the field of neurogastroenterology and motility to contemplate again on the impact of gender factors on FGIDs.
Financial support: This work was supported Wonkwang University 2014 to Jaehoon Jahng.

\section{Conflicts of interest: None.}

\section{References}

1. Kim YS, Kim N, Kim GH. Sex and gender differences in gastroesophageal reflux disease. J Neurogastroenterol Motil 2016;22:575-588

2. Clayton JA, Collins FS. Policy: NIH to balance sex in cell and animal studies. Nature 2014;509:282-283.

3. NIH policy and guidelines on the inclusion of women and minorities as subjects in clinical research - amended, October, 2001. Available from URL: http:/grants.nih.gov/grants/funding/women_min/guidelines_ amended_10_2001.htm (accessed 6 December, 2016).

4. Moussaoui N, Jacobs JP, Larauche M, et al. Chronic early-life stress in rat pups alters basal corticosterone, intestinal permeability, and fecal microbiota at weaning: influence of sex. J Neurogastroenterol Motil 2017;23:135-144.

5. Gendered Innovations. Available from URL: https://genderedinnovations.stanford.edu/ (accessed 6 December, 2016).

6. Houghton LA, Heitkemper M, Crowell M, et al. Age, gender and women's health and the patient. Gastroenterology 2016;150:1332-1343. e4.

7. Payne S. Sex, gender, and irritable bowel syndrome: making the connections. Gend Med 2004;1:18-28.

8. Toner BB, Akman D. Gender role and irritable bowel syndrome: literature review and hypothesis. Am J Gastroenterol 2000;95:11-16. 